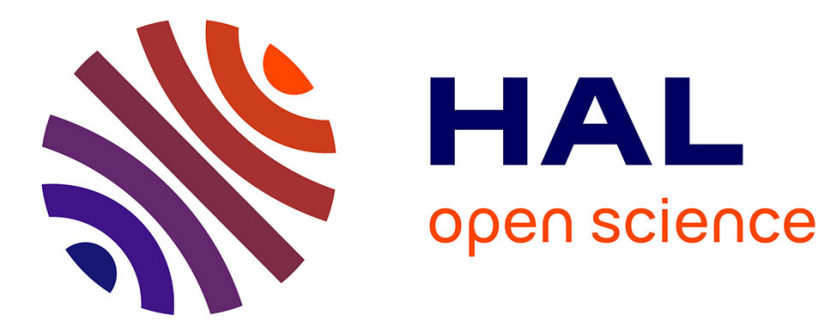

\title{
Thirty years of hydrogen spectroscopy in Paris
}

François Biraben, Lucile Julien, François Nez

\section{To cite this version:}

François Biraben, Lucile Julien, François Nez. Thirty years of hydrogen spectroscopy in Paris. Applied Physics B - Laser and Optics, 2017, 123, pp.50. 10.1007/s00340-016-6619-1 . hal-01457882

\section{HAL Id: hal-01457882 https://hal.sorbonne-universite.fr/hal-01457882}

Submitted on 6 Feb 2017

HAL is a multi-disciplinary open access archive for the deposit and dissemination of scientific research documents, whether they are published or not. The documents may come from teaching and research institutions in France or abroad, or from public or private research centers.
L'archive ouverte pluridisciplinaire HAL, est destinée au dépôt et à la diffusion de documents scientifiques de niveau recherche, publiés ou non, émanant des établissements d'enseignement et de recherche français ou étrangers, des laboratoires publics ou privés. 


\title{
Thirty years of hydrogen spectroscopy in Paris
}

\author{
François Biraben $^{1} \cdot$ Lucile Julien $^{1} \cdot$ François Nez $^{1}$ (D)
}

Received: 12 September 2016 / Accepted: 15 December 2016

(C) Springer-Verlag Berlin Heidelberg 2017

\begin{abstract}
This paper gives a review of the experiments performed since the 1980s at the Laboratoire Kastler Brossel in Paris on two-photon spectroscopy of atomic hydrogen. Firstly devoted to the $2 \mathrm{~S}-n \mathrm{~S}$ and $2 \mathrm{~S}-n \mathrm{D}$ transitions, they are currently running on the $1 \mathrm{~S}-3 \mathrm{~S}$ transition at 205 $\mathrm{nm}$. During all that time, they were inspired by the plentiful ideas proposed by Ted Hänsch and were complementary with the measurements developed in parallel in his groups.
\end{abstract}

\section{Introduction}

Hydrogen spectroscopy in Paris is a long story which began in 1983. One of us (FB) decided to use the tunable cw monomode laser he had himself developed [1] to excite two-photon transitions in atomic hydrogen. At this time, Ted Hänsch's work on hydrogen - the simplest and the most fascinating of the atoms-were worldwide known: in particular, the first observation of the $2 \mathrm{~S}$ Lamb shift in an optical spectrum recorded by saturated absorption [2] and the one of the $1 \mathrm{~S}-2 \mathrm{~S}$ two-photon transitions using a pulsed laser [3].

The Paris idea was then to choose other transitions than the $1 \mathrm{~S}-2 \mathrm{~S}$ one, actually transitions starting from the $2 \mathrm{~S}$ metastable state, to excite Rydberg S or D states. After

This article is part of the topical collection "Enlightening the World with the Laser" - Honoring T. W. Hänsch guest edited by Tilman Esslinger, Nathalie Picqué, and Thomas Udem.

François Nez

francois.nez@1kb.upmc.fr

1 Laboratoire Kastler Brossel, UPMC-Sorbonne Universités, CNRS, Collège de France, ENS-PSL Research University, 4 Place Jussieu, 75005 Paris, France several years, we also moved to the $1 \mathrm{~S}-3 \mathrm{~S}$ transition, a transition from the ground state, which is nowadays still under study in our group.

We show in this paper how Paris works in atomic hydrogen have been stimulated by the ones of Ted Hänsch's groups, first in Stanford and then in Garching, and how they developed in a context where an healthy competition turned often into collaboration and joint efforts for a common goal.

\section{The beginning of the Paris story}

At the beginning, we (FB and $\mathrm{LJ}$ ) were then only two people on the hydrogen project in Paris, with the scientific support of Bernard Cagnac who founded a few years before in our laboratory a group devoted to Doppler-free two-photon spectroscopy. He himself had pointed out for the first time the advantage of this spectroscopy for the study of the 1S-2S transition in hydrogen [4] but without undertaking such an experiment since Ted Hänsch seized this subject very quickly. It is why we decided to study other hydrogen lines.

One of us (F.B.) had already a very good expertise in high-resolution spectroscopy, having studied two-photon transitions in sodium [5] and rare gas atoms [6, 7]. The other one (L.J.) had previously measured atomic structures and Lamb shifts in excited hydrogen states by an anticrossing method [8].

Our target was to excite the $n \geq 8$ states because the wavelength range needed $730-778 \mathrm{~nm}$ was easily obtained with our homemade dye laser.

In a first step, we had to build a $2 \mathrm{~S}$ metastable atomic beam, which was obtained by the following method: molecular hydrogen is dissociated by a RF discharge in a 

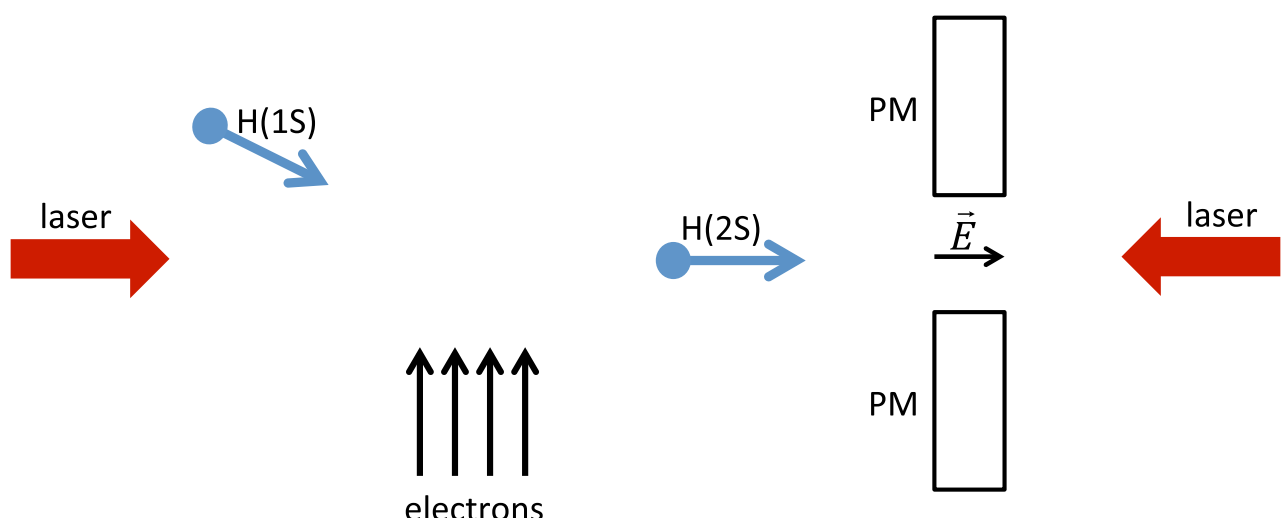

Fig. 1 Geometry of the two-photon excitation for the observation of $2 \mathrm{~S}-n \mathrm{~S} / n \mathrm{D}$ signals. After interaction with the two counter-propagating laser beams, the remaining $2 \mathrm{~S}$ atoms are detected through electric quenching and Lyman alpha fluorescence

pyrex tube; an effusive $1 \mathrm{~S}$ beam flows into a first vacuum chamber through a Teflon nozzle; $2 \mathrm{~S}$ state is excited by electronic bombardment which bends the atomic beam and then makes it collinear with the two laser beams used for the two-photon excitation. The grid of the electron gun creates an equipotential volume around the metastable beam to shield it from quenching electric fields. The interaction between atoms and laser beams takes place in a second vacuum chamber delimited by two holes $96 \mathrm{~cm}$ apart from each other. The atoms remaining in the $2 \mathrm{~S}$ state are detected in a third chamber where two electrodes quench the $2 \mathrm{~S}$ state and the resulting Lyman- $\alpha$ fluorescence is detected. The two-photon transition signal is recorded through the decrease of the number of metastable atoms at the end of the beam. The schematized geometry of the excitation is shown in Fig. 1.

To enhance the two-photon excitation probability, the whole metastable beam was placed inside a Fabry-Perot cavity, the length of which was locked on the laser frequency by monitoring the reflected polarization, using the famous method proposed by Hänsch and Couillaud [9].

The search for $n=8$ signal was not straightforward. Our laser frequency was scanned by locking it to a pressure swept Fabry-Perot cavity and known by comparison with a reference cavity whose length was not perfectly determined. Moreover, the Rydberg constant was actually known with a poorer exactness than believed, so that we did not look for our resonance at the correct frequency. After several months of efforts and changing the curvature of the enhancing cavity mirrors to optimize the excitation probability along the beam, we finally obtained signals (see Fig. 2) in both hydrogen and deuterium and were able to perform a preliminary measurement of the $n=8$ isotopic shift [10]. The way to absolute measurements was now open for us.

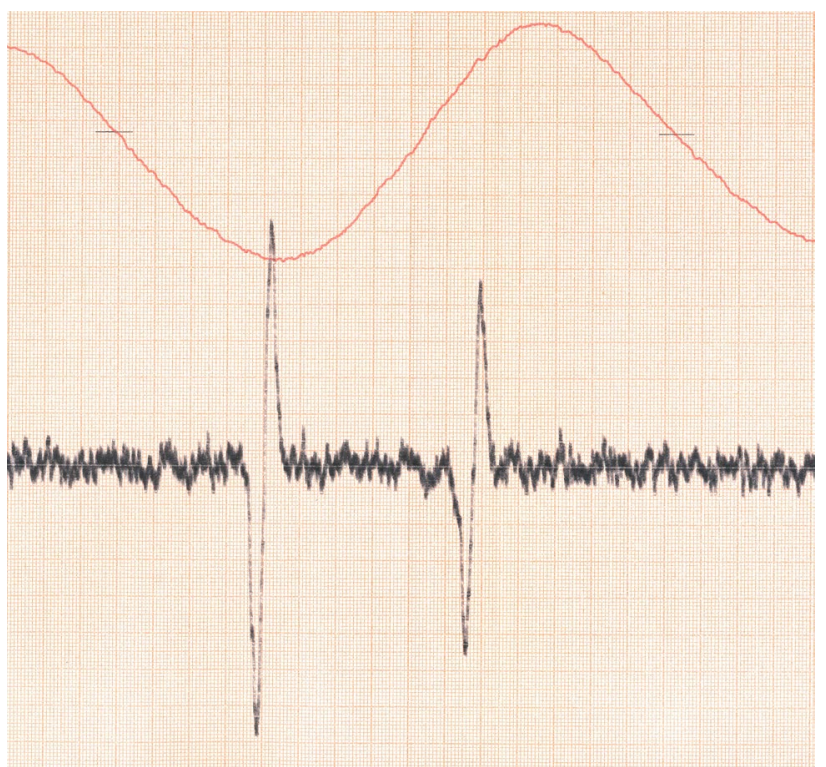

Fig. 2 2S-8D signal recorded on November 9, 1984. The two fine structure components of the $8 \mathrm{D}$ level are visible, separated by about $57 \mathrm{MHz}$. The laser frequency is modulated and the $2 \mathrm{~S}$ signal demodulated by a lock-in amplifier. The red trace is the transmission of a FP cavity used for the calibration, having a low finesse at this wavelength

\section{Wavelength measurements and first determinations of the Rydberg constant}

The largest signal amplitude was the $2 \mathrm{~S}_{1 / 2}(F=1) \rightarrow 8 \mathrm{D}_{5 / 2}$ one, with a $10 \%$ decrease of the metastable beam intensity, and an experimental width of 1.4 $\mathrm{MHz}$ (relative linewidth $1.8 \times 10^{-9}$ ), which was at that time the narrowest one obtained in hydrogen. This width has to be compared to the $550 \mathrm{kHz}$ natural width of the 8D states. The main broadening and shift effects were the light 
shift, the saturation of the $2 \mathrm{~S}$ depopulation, the secondorder Doppler effect and the finite transit time of the atoms in the laser beams.

Rapidly, we observed not only the $2 \mathrm{~S}-8 \mathrm{D}$ transitions in $\mathrm{H}$ and $\mathrm{D}$ at $778 \mathrm{~nm}$ but also the 2S-10D ones in $\mathrm{H}$ at 760 $\mathrm{nm}$, and we undertook to determine the Rydberg constant from the measurement of the three $2 \mathrm{~S}-n \mathrm{D}_{5 / 2}$ transition wavelengths.

The most recent determinations of the Rydberg constant were previously deduced either from the one-photon $2 \mathrm{~S}-3 \mathrm{P}$ and 2P-3S Balmer- $\alpha$ transitions [11-13], or from the 1S-2S two-photon transition, both methods in which Ted Hänsch took a pioneer role. The first one has the disadvantage of being limited to about $10^{-9}$ by the natural width of the $\mathrm{P}$ level involved. The second one was much more promising, but needs the development of a tunable laser source at $243 \mathrm{~nm}$. Although the $1 \mathrm{~S}-2 \mathrm{~S}$ signal had been already observed with a relative linewidth of $5 \times 10^{-9}$ using $\mathrm{cw}$ excitation [14], the first wavelength measurement had been performed with a pulsed laser and a much broader signal [15].

Compared to the $1 \mathrm{~S}-2 \mathrm{~S}$ transition, the $2 \mathrm{~S}-n \mathrm{D}$ ones ( $n \geq 8$ or 10 ) were limited by a much larger natural width but were competitive at that time for the determination of the Rydberg constant, since the $2 \mathrm{~S}$ Lamb shift was precisely measured by RF spectroscopy [16, 17].

We measured our transition wavelengths [18] by interferometric comparison with an iodine-stabilized heliumneon laser at $633 \mathrm{~nm}$. The wavelength of this laser was known at $2 \times 10^{-10}$ since it had been itself compared to one of the "Institut National de Métrologie." We deduced our first value of the Rydberg constant with an uncertainty of $5.2 \times 10^{-10}$, but this value was in slight disagreement with the other ones published. As often in such a situation, such a discrepancy is uncomfortable for a new team in the field, but is a strong encouragement to pursue it.

During the following years, we studied also the $2 \mathrm{~S}-8 \mathrm{~S}$ transition and performed a detailed study of the line profiles taking into account for each transition all shifting and broadening effects for various values of the excitation power. Calculated profiles were then fitted to experimental signals in order to determine the line positions corrected from these effects. Examples of such fits are shown in Fig. 3 for the $2 S_{1 / 2}-8 S_{1 / 2}$ and $2 S_{1 / 2}-8 D_{5 / 2}$ transitions. Here the quenching field at the end of the atomic beam is modulated and the $2 \mathrm{~S}$ signal demodulated by a lock-in amplifier. A good agreement is observed for both transitions, even if their line profiles have different asymmetries.

During this period, we also worked to reduce systematic effects and were able to extend our method to higher $n$ levels. For that purpose, our interaction region was drastically shielded from stray electric fields by painting the walls of the vacuum chamber with Aquadag, a graphite liquid mixture: we were then able to record 2S-20D two-photon signals and to estimate an upper limit for the electric field experienced by the atoms $(\leq 2 \mathrm{mV} / \mathrm{cm})$, and for the resulting Stark effect on our signals [19]. Another improvement was to measure the velocity distribution of our metastable atomic beam, by probing the $2 \mathrm{~S}-3 \mathrm{P}$ Balmer- $\alpha$ one-photon transition with a collinear $656 \mathrm{~nm}$ laser beam [20].

In 1989, our result for the Rydberg constant with an uncertainty of $1.7 \times 10^{-10}$ was mainly limited by the standard He-Ne laser [21]. During the same time, a good agreement of the results obtained on other transitions in hydrogen was found [22-24], especially after elimination of chirping effects in pulsed amplifiers, previously used for the $1 \mathrm{~S}-2 \mathrm{~S}$ excitation.

However, in these measurements, as in others, our laser wavelength was measured by interferometric comparison to the one of the standard lasers using the so-called virtual mirrors method, and the resulting uncertainty arose both from the knowledge of the reference laser frequency and from the comparison method itself. The further reduction of the uncertainty needed to abandon wavelength measurement for direct frequency comparisons.

Let us also point out here that in 1990 our homemade excitation dye laser was turned into a Ti:sapphire laser (TiSa), perfectly suitable for our $730-778 \mathrm{~nm}$ range and much more convenient in terms of stability, efficiency and easiness of running.

\section{Direct comparison of two optical frequencies}

As explained in the following, absolute optical frequency measurements were a quite complicated issue in the early 1990s. On the other hand, the determination of the Rydberg constant from the study of an optical transition was limited by the knowledge of the Lamb shifts of the levels involved. Since in atomic hydrogen the ratio between transition frequencies is very close to simple rational fractions, both Ted's group in Garching and our Paris group decided to complete their experimental setup in order to directly compare transition frequencies with a ratio close to 4:1: the $1 \mathrm{~S}-2 \mathrm{~S}$ and $2 \mathrm{~S}-4 \mathrm{~S} / 4 \mathrm{D}$ transitions in Garching, the $1 \mathrm{~S}-3 \mathrm{~S}$ and $2 \mathrm{~S}-6 \mathrm{~S} / 6 \mathrm{D}$ ones in Paris.

In Garching, a new $2 \mathrm{~S}$ metastable beam was then developed with a Ti:sapphire laser at $972 \mathrm{~nm}$ for the atom excitation. In a similar way as in Paris, residual $2 \mathrm{~S}$ atoms were detected at the end of the interaction region. The numerical calculation of the 2S-4S/4D signal profile was an opportunity for a fruitful collaboration between our two groups [25].

In Paris, the experiment was not easy to achieve, but during one year we benefited from the precious help of Derek Stacey, who had an expertise in the UV domain since he 
Fig. $32 \mathrm{~S}_{1 / 2}-8 \mathrm{~S}_{1 / 2}$ (upper part) and $2 \mathrm{~S}_{1 / 2}-8 \mathrm{D}_{5 / 2}$ (lower part) signals recorded in 1996, with fitted calculated profiles superimposed. Vertical scale number of $2 \mathrm{~S}$ atoms recorded after demodulation (see text) by a lock-in and offset. The signal amplitudes are, respectively, 4.4 and $21.0 \%$ of the $2 \mathrm{~S}$ population. Horizontal scale frequency of the acoustooptic modulator used to scan the excitation laser. The different asymmetries of the lines arise from the respective weight of saturation and light shift. The saturation is almost negligible for the $8 \mathrm{~S}$ line and important for the $8 \mathrm{D}$ one
NIVEAU $8 \mathrm{~S} 1 / 2(\mathrm{~F}=1)$

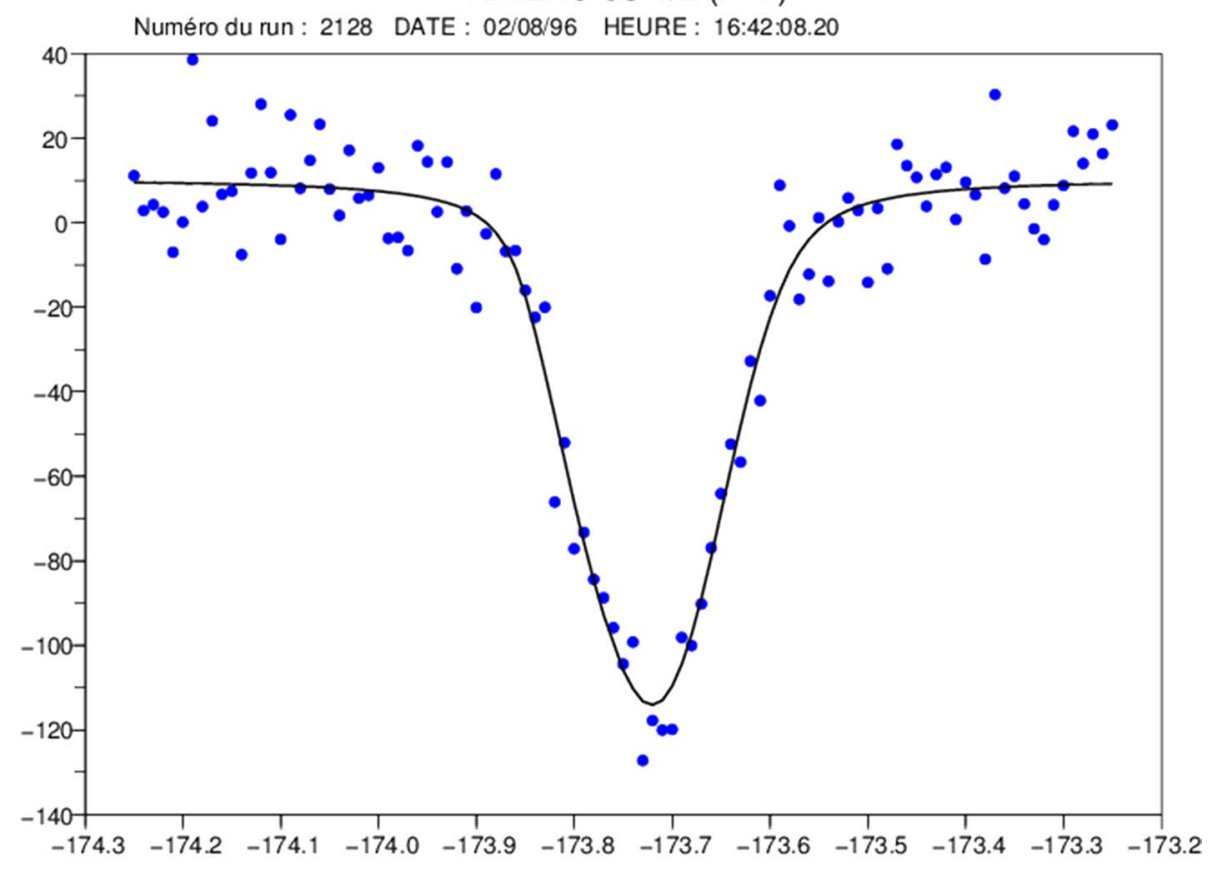

NIVEAU 8D 5/2 ( $F=1)$

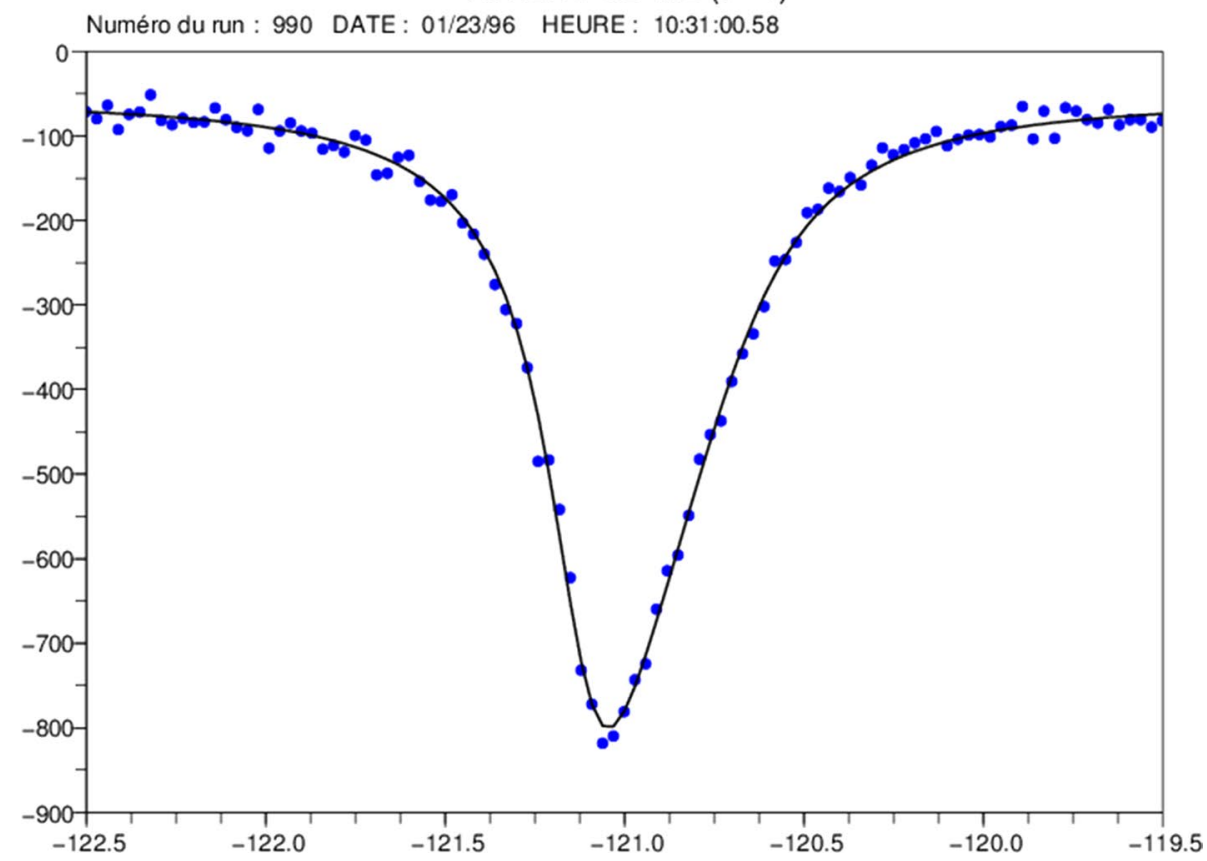

had studied the 1S-2S transition in the Oxford group [27]. A $1 \mathrm{~S}$ beam was build and we had to develop a new laser source at $205 \mathrm{~nm}$. For that purpose we chose to perform two successive frequency doubling stages of the Ti-sapphire radiation at $820 \mathrm{~nm}$ used for the $2 \mathrm{~S}-6 \mathrm{~S} / 6 \mathrm{D}$ excitation. The first doubling in an enhancement external cavity was quite efficient, and we obtained more than $400 \mathrm{~mW}$ at 410 nm with our LBO crystal [28]. However, the second doubling step in a BBO crystal was far more challenging since
$205 \mathrm{~nm}$ is the shortest wavelength which can be generated by SHG in such a crystal and because of photochemical reactions on the crystal faces and photorefractive effects in its bulk. We finally got only about $1 \mathrm{~mW}$ in a quasi-continuous mode where the enhancement cavity length is modulated [29].

Compared to the $1 \mathrm{~S}-2 \mathrm{~S}$ transition, the $1 \mathrm{~S}-3 \mathrm{~S}$ one is much broader and the signal much smaller, so that it was not straightforward for us to see the signal. It was finally 
observed in 1995 . On the other hand the $2 \mathrm{~S}-6 \mathrm{~S} / 6 \mathrm{D}$ transitions are much narrower than the $2 \mathrm{~S}-4 \mathrm{~S} / 4 \mathrm{D}$ ones and, following the Garching and Yale groups [25, 26], we were able to determine the $1 \mathrm{~S}$ Lamb shift with an uncertainty of $46 \mathrm{kHz}$ [30], using the experimental value of the $2 \mathrm{~S}$ one. The results obtained by the three groups were in good agreement with each other and, with comparable uncertainties, they notably improved the knowledge of the $1 \mathrm{~S} \mathrm{Lamb}$ shift. However, they were limited by the precision of the $2 S$ Lamb shift, measured in the RF domain. To go further, it was necessary to perform the absolute frequency measurement of two different hydrogen optical frequencies.

\section{Absolute optical frequency measurements in Paris}

The first absolute frequency measurement of an hydrogen transition was the one of the $2 \mathrm{~S}-8 \mathrm{D}$ transitions [31], performed in our group during the $\mathrm{PhD}$ thesis of the third of us (F.N., also called F2). At that time, such a measurement needed to have a good frequency reference in the laboratory and a convenient frequency chain to compare the frequency to be measured to the one of the reference.

\subsection{Frequency references}

Our wavelength measurements had been previously performed by interferometric comparison with an $I_{2}$-stabilized $\mathrm{He}-\mathrm{Ne}$ laser at $633 \mathrm{~nm}$. This reference laser had been itself calibrated by comparison with the Bureau International des Poids et Mesures (BIPM) standard lasers through an intermediate laser, so that its frequency was known with an uncertainty of $1.6 \times 10^{-10}$.

In order to implement direct frequency measurements in hydrogen, we decided to look for new references around $778 \mathrm{~nm}$, the wavelength of the $2 \mathrm{~S}-8 \mathrm{~S} / \mathrm{nD}$ two-photon transitions. After a tentative work with the hyperfine components of the $\mathrm{IBr}$ molecule located in this frequency range [32], we finally chose the ones of the 5S-5D two-photon transitions in $\mathrm{Rb}$ [33]. Laser diodes could easily be locked to these transitions, giving a new optical frequency standard at $778 \mathrm{~nm}$. Its frequency was measured, thanks to a collaboration with the Laboratoire Primaire du Temps et des Fréquences (LPTF, presently LNE-SYRTE) and the BIPM, with an uncertainty of $2 \mathrm{kHz}$ [34]. This frequency standard with high metrological features [35] is still used in our hydrogen setup.

At the same time, after several years of effort and thanks to the tenacity of B. Cagnac, a 3-km-long optical fiber has been placed underground between our laboratory and the LPTF (LNE-SYRTE). This opened up the possibility to transfer optical frequencies between our two laboratories with an accuracy of a few $\mathrm{Hz}$ [36] and then to connect our experiment to the primary frequency standard, the $\mathrm{Cs}$ atomic clock.

\subsection{Frequency chains}

The first frequency chain built in Paris to measure the 2S-8S/8D transitions of hydrogen used actually two frequency standards: the $I_{2}$-stabilized $\mathrm{He}-\mathrm{Ne}$ laser at 473 $\mathrm{THz}(633 \mathrm{~nm})$ and the $\mathrm{CH}_{4}$-stabilized $\mathrm{He}-\mathrm{Ne}$ laser at 88 $\mathrm{THz}(3.39 \mu \mathrm{m})$. It took advantage of the quasi-coincidence of the frequency difference between these two frequency standards with the one of our transitions ( 2 photons at 385 $\mathrm{THz}$ ). Both standard frequencies were precisely known, thanks to recent calibrations done at the LPTF, and the 89 $\mathrm{GHz}$ frequency gap was measured in our laboratory firstly using a Fabry-Perot cavity [37], secondly using a Schottky diode which means that we realized for the first time a direct link to the Cs clock without any interferometry [31]. The Rydberg constant was deduced with an uncertainty of $2.2 \times 10^{-11}$.

In the following, this result could be improved to $9 \times 10^{-12}$ thanks to the $\mathrm{Rb}$ frequency standard and the optical fiber discussed above, and the measurement was extended to deuterium, giving a new determination of the 2S Lamb shift in this atom [38].

As the direct frequency comparison with our $\mathrm{Rb}$ standard was only possible for the $2 \mathrm{~S}-8 \mathrm{~S} / 8 \mathrm{D}$ transitions, we were obliged to build-up again a specific frequency chain to measure other transitions in hydrogen. As a new target, we chose the $n=12$ levels which are very sensitive to stray electric fields (the quadratic Stark effect varies as $n^{7}$ ) and then give complementary information to possible systematic effects on the $n=8$ transitions. This choice was motivated by the possibility to compare the transition frequency at $799 \mathrm{THz}$ ( $2 \mathrm{TiSa}$ photons at $750 \mathrm{~nm}$ to excite the $n=12$ levels in $\mathrm{H}$ ) to twice the one of the $\mathrm{Rb}$ standard at $385 \mathrm{THz}$, using a standard $\mathrm{OsO}_{4}$-stabilized $\mathrm{CO}_{2}$ laser at $29 \mathrm{THz}(\approx 10$ $\mu \mathrm{m})$ to measure the gap. One has indeed:

$799 \mathrm{THz}=2 \times 385 \mathrm{THz}+29 \mathrm{THz}$

In this new chain, we used an optical frequency divider to reduce by a factor 2 the frequency difference between our TiSa laser and the Rb standard, as suggested for the first time by Hänsch et al. [39].

The experiment was simultaneously carried out in our laboratory and in the LPTF, thanks to our link by a double optical fiber. An auxiliary $809 \mathrm{~nm}$ radiation $(370.5 \mathrm{THz}$ ) delivered by a laser diode in our laboratory was sent to the LPTF to be compared to the frequency difference between a laser diode at $750 \mathrm{~nm}(385 \mathrm{THz})$ and the $\mathrm{CO}_{2} / \mathrm{OsO}_{4}$ laser. A radiation at $750 \mathrm{~nm}$ was also send to our laboratory to be compared to our TiSa laser, whose frequency was mixed 
with the one of the auxiliary laser diodes at $809 \mathrm{~nm}$. The frequency sum obtained was compared to twice the one of the $\mathrm{Rb}$ standard. The two following equations were then realized, respectively, in LPTF and in LKB:

$370.5 \mathrm{THz}($ auxiliaryDL) $=385 \mathrm{THz}-29 \mathrm{THz} / 2$

$$
\begin{array}{r}
399.5 \mathrm{THz}(\mathrm{Ti}-\mathrm{Sa})+370.5 \mathrm{THz} \\
(\text { auxiliaryDL })=2 \times 385 \mathrm{THz}
\end{array}
$$

The four 2S-12D transitions in hydrogen and deuterium were measured and a careful analysis of the line profiles was carried out, including various systematic effects, in particular the Stark effect. Using this result and the ones obtained by other groups, especially the Garching one on the $1 \mathrm{~S}-2 \mathrm{~S}$ transition, the Rydberg constant could be deduced with an uncertainty lower than $8 \times 10^{-12}[40,41]$.

\subsection{Frequency combs}

As in Paris, the study of hydrogen transitions in Ted Hänsch's group, first in Stanford and then in Garching, needed during the same period successively the design of an auxiliary standard laser [42], the development of a frequency chain with a frequency divider [43], the use of a transportable calibrated standard [44] and finally a direct link to a metrology institute through an optical fiber [45]. All these means were used to measure the $1 \mathrm{~S}-2 \mathrm{~S}$ frequency with an increasing and unbeatable precision.

Moreover, during the same time occurred the so-called frequency comb revolution, the main father of which is Ted Hänsch, rewarded by the well-deserved Nobel Prize jointly awarded to John Hall and Ted Hänsch in 2005. The life in our laboratories was totally changed. No more need of complicated frequency chains depending on the transition to be measured: a fs laser coupled to a photonic fiber was the unique instrument needed to measure optical frequencies, allowing one to compare them directly to the Cs frequency standard. What we dreamed about was realized.

Of course, the $1 \mathrm{~S}-2 \mathrm{~S}$ transition was the first one in hydrogen to be measured in this new way [46], and other improvements followed $[47,48]$. For us, it was time to leave $2 \mathrm{~S}-n \mathrm{~S} / n \mathrm{D}$ and focus on $1 \mathrm{~S}-3 \mathrm{~S}$.

\section{From $2 \mathrm{~S}-n \mathrm{~S} / n \mathrm{D}$ to $1 \mathrm{~S}-3 \mathrm{~S}$ spectroscopy}

At the turn of the century, it seemed to us that it was no more possible for us to push further our precision on the transitions starting from the $2 \mathrm{~S}$ metastable state. Let us point out the advantages and the limitations of these transitions.

They are in a frequency range easy to reach with a TiSa laser and have small natural widths of a few hundreds of
$\mathrm{kHz}$. The stray electric field was reduced at best and estimated by recording the transitions toward $n=20$ levels, and the velocity distribution measured (see above).

However, the number of metastable atoms in our atomic beam was small $2 \times 10^{6}$ at/s being a limiting factor for our signal-to-noise ratio. We then used a high laser power to excite the transitions: up to $150 \mathrm{~W}$ in each direction of propagation inside the enhancement cavity. Due to the conjugated effect of saturation of the $2 \mathrm{~S}$ depopulation and light shifts, the line profiles are asymmetric (see Fig. 3) but well understood by numerical simulations. Extrapolation to null power of the center of the line is performed using a procedure detailed in [41].

The features of the 1S-3S transition are quite different. The number of $1 \mathrm{~S}$ atoms is about eight orders of magnitude larger than the metastable one. The natural width of $1 \mathrm{MHz}$ is similar, but the excitation wavelength which lies in the UV range $(205 \mathrm{~nm})$ is responsible for a better quality factor for the line. The laser power is only a few milliwatts and the light shift much smaller. Moreover, the residual Stark shift effect is negligible.

However, since we have no tunable laser source to probe the velocity distribution of the $1 \mathrm{~S}$ beam through a one-photon transition, we have no simple way to determine the second-order Doppler effect which shifts the line by $-v^{2} / 2 c^{2}$, almost $150 \mathrm{kHz}$. This is why we have proposed and implemented an original method allowing to measure this shift [49] in our thermal atomic beam. The basic idea is to apply a transverse magnetic field to the atoms, inducing a quadratic motional Stark effect varying also as $v^{2}$ and able to partially compensate the second-order Doppler effect near a 3S-3P anticrossing [50].

With this method and using a frequency comb, we measured for the first time the absolute frequency of the $1 \mathrm{~S}-3 \mathrm{~S}$ transition [51], with an uncertainty of $13 \mathrm{kHz}$ $\left(4.5 \times 10^{-12}\right)$. After the measurement of the $1 \mathrm{~S}-2 \mathrm{~S}$ transition in Garching, our result was in 2010 the second most precise one of the optical transitions in hydrogen. This experiment is still being improved. We have in particular developed a new laser source at $205 \mathrm{~nm}$, by frequency mixing of two radiations at 266 and $894 \mathrm{~nm}$, respectively generated by frequency doubling of a Verdi laser (Coherent Inc.) at $532 \mathrm{~nm}$ and by our TiSa laser. By this method, we obtain up to $15 \mathrm{~mW}$ of $\mathrm{cw}$ operation at $205 \mathrm{~nm}$ [52].

In 2013, the 1S-3S signal was observed with a signal-to noise ratio up to 170 after $3.5 \mathrm{~h}$ of integration, and with a width of about $1.5 \mathrm{MHz}$, to be compared to the $1 \mathrm{MHz}$ natural width of the line. The absolute frequencies of the two radiations at $532 \mathrm{~nm}$ and $894 \mathrm{~nm}$ were measured with an optical frequency comb referenced to the Cs clock of the LNE-SYRTE laboratory. The statistical uncertainty on the deduced transition frequency has been significantly improved to reach a value of $2.2 \mathrm{kHz}$ [53]. However, a 
complete analysis of systematic shifts of the line, in particular collisional shifts, was still needed to determine the $1 \mathrm{~S}-3 \mathrm{~S}$ transition frequency.

This problem is presently under study. Our experiment is in progress and a recent signal is recorded in Fig. 4.

\section{Hydrogen spectroscopy and the Rydberg constant}

When we began the study of $2 \mathrm{~S}-n \mathrm{D}$ transitions during the 1980s, our first goal was the determination of the Rydberg constant. It was simply deduced from our transition frequencies, using the experimental value of the $2 \mathrm{~S}$ Lamb shift. Such a method was limited to an uncertainty of $1.2 \times 10^{-11}$ due to the $2 \mathrm{~S}$ Lamb shift measurement.

To go further in precision, two different optical transitions were needed, which were found in the $1 \mathrm{~S}-2 \mathrm{~S}$ and $2 \mathrm{~S}-8 \mathrm{D}$ transitions. As the quantity $L(1 \mathrm{~S})-8 L(2 \mathrm{~S})$ had been precisely calculated [54-56], the comparison of the two optical frequency intervals allows the determination of the Rydberg constant with an uncertainty of $8.6 \times 10^{-12}$. The advantage of this method is that it needs neither the $2 \mathrm{~S}$ Lamb shift nor the proton radius and is applicable both to hydrogen and deuterium.

Finally, the most precise value of the Rydberg constant is given by the least squares adjustment of the fundamental constants performed by the Committee on Data for Science and Technology (CODATA). It takes into account data concerning all the fundamental constants, but is limited to spectroscopic data on hydrogen (e-p) only and e-p diffusion experiments. The uncertainty deduced for the Rydberg constant by the last adjustment is $5.5 \times 10^{-12}$ [57] resulting mostly from the uncertainties obtained by the Paris and the Garching groups on the $2 \mathrm{~S}-n \mathrm{~S} / n \mathrm{D}$ and $1 \mathrm{~S}-2 \mathrm{~S}$ transitions.

In the same manner as for the Rydberg constant, hydrogen $1 \mathrm{~S}$ and $2 \mathrm{~S}$ Lamb shifts can be deduced from the same measurements, using the scaling law quoted above. The value of the $2 \mathrm{~S}-2 \mathrm{P}$ interval obtained is more precise than the one given by direct microwave spectroscopy. The $1 \mathrm{~S}$ Lamb shift with an uncertainty of $24 \mathrm{kHz}$ only would give a test of the QED two-loop corrections in QED if the proton charge radius $r_{\mathrm{p}}$ was perfectly known. On the other hand, the QED calculations are a way to determine $r_{\mathrm{p}}$, which is complementary with the e-p scattering experiments.

\section{The hydrogen atom, a still hot topic}

At the end of the 1990s, it was then clear that the proton radius became a limiting factor to test theoretical predictions of QED on the hydrogen atom. It is why we joined in 1998 the international collaboration, now called CREMA,

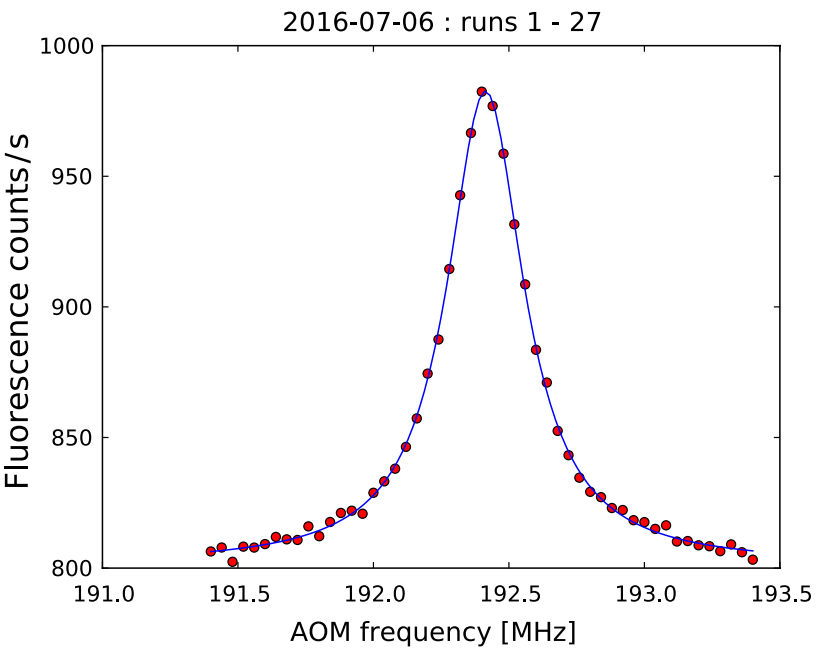

Fig. 4 1S-3S signal recorded on July 6, 2016. The excitation laser at $205 \mathrm{~nm}$ is swept through an acousto-optic modulator. The integration time is here $4.5 \mathrm{~h}$ and the signal linewidth $1.36 \mathrm{MHz}$ in atomic frequency units. The signal background is mainly due to stray fluorescence induced by the UV excitation light

constituted to determine $r_{\mathrm{p}}$ from $2 \mathrm{~S}-2 \mathrm{P}$ spectroscopy of muonic hydrogen at the Paul Scherrer Institute (PSI) in Switzerland. The Paris and the Garching groups were active together in this new adventure, which is not closed at the present time.

The idea was to measure the $2 \mathrm{~S}-2 \mathrm{P}$ Lamb shift interval in muonic hydrogen, an atom made of a proton and a negative muon $\left(\mu^{-}\right)$. As the muon is 207 times more massive than the electron, the correction due to the finite size of the nucleus is largely enhanced. Indeed, the sensitivity of the frequency of the $2 \mathrm{~S}-2 \mathrm{P}$ transition to $r_{\mathrm{p}}$ in such a spectroscopy at the $10^{-6}$ level is enough to get a determination of $r_{\mathrm{p}} 10$ times better than the best determination of $r_{\mathrm{p}}$ from the electronic hydrogen spectroscopy and quantum electrodynamics.

The principle of the experiment can be summarized in three steps: production of muonic hydrogen $(\mu-\mathrm{p})$ atoms in the metastable $2 \mathrm{~S}$ state, excitation of the $2 \mathrm{~S}-2 \mathrm{P}$ transition with a laser at $6 \mu \mathrm{m}$ and then detection of the $2 \mathrm{P}-1 \mathrm{~S}$ fluorescence photon at $2 \mathrm{keV}$. Even if it looks simple, each step was challenging. The source of metastable atoms has been realized thanks to the expertise of F. Kottmann, D. Taqqu and R. Pohl. Members of the Paris group took part in the development of the laser chain which produced the $6 \mu \mathrm{m}$ laser pulse in collaboration with A. Antognini. After three unsuccessful beam times (2002, 2003, 2007), in 2009, we finally observed two transitions of muonic hydrogen and three of muonic deuterium [58-60], but they were not at the frequency predicted by the theory. This was the beginning of the so-called proton size puzzle which has triggered and stimulated a lot of activities in our community 
but also outside of it, both theoretical and experimental ones.

On the side of experiments in hydrogen atom, a new RF measurement of the $2 \mathrm{~S}-2 \mathrm{P}$ hydrogen interval is currently in progress in the group of Hessels [61] while the Paris and Garching groups pursue their efforts to improve their optical measurements: in the one-photon $2 \mathrm{~S}-4 \mathrm{P}$ transition [62] (cw laser in Garching) and in the two-photon 1S-3S transition [53, 63] (pulsed laser in Garching and $\mathrm{cw}$ laser in Paris). At the level of precision needed to clarify the proton puzzle, all stray shifting effects have to be carefully studied, including quantum cross-damping interference effects [64-66].

New results are looked forward in the near future, not only on the above hydrogen experiments, but also on hydrogenic systems [67-70].

\section{Conclusion}

This review of more than thirty years of hydrogen spectroscopy in Paris and also in Ted Hänsch's groups in Stanford and Garching shows how the study of this simple atom has stimulated the development of laser technology and new frequency measurement methods in parallel with QED calculations.

Thanks to the development of frequency combs, ultrastable cavities and laser references, the optical part of an hydrogen experiment is almost no more an issue, except for the lack of a powerful $\mathrm{cw} 121 \mathrm{~nm}$ laser to optically cool atomic hydrogen, even if $1 \mathrm{~S}-2 \mathrm{P}$ spectroscopy has been already performed with a low power source in Ted Hänsch's group [71]. The last experimental frontier in hydrogen spectroscopy is certainly the atomic source: hydrogen spectroscopy is still performed on atomic beams.

The other frontier to overcome is in the perturbative QED calculations. Nowadays, the $1 \mathrm{~S}-2 \mathrm{~S}$ transition frequency is measured with an uncertainty of $10 \mathrm{~Hz}$ that is 250 times smaller than the theoretical uncertainty!

From the beginning of quantum mechanics, the hydrogen atom is a source of new discoveries in physics and can be considered as "the Rosetta stone of modern Physics" as pointed out in a paper of Schawlow et al. [72]. Coming back to the Paris adventure, two of us who were at the beginning of this story are now retired, although still active in research. We did not imagine that after more than thirty years, the hydrogen atom would be always so fascinating and rich of secrets still to discover.

Acknowledgements We are pleased to acknowledge here all people who worked with us on our "hydrogen experiments" in Paris: JeanClaude Garreau, Sophie Bourzeix, Béatrice Chatel (De Beauvoir), Gaëtan Hagel, Olivier Arnoult, Sandrine Galtier and Hélène Fleurbaey as Ph.D. students, Mark D. Plimmer, F. de Tomasi and L. Jozefowski as post-docs and our colleagues M. Allegrini, D.N. Stacey and C. Schwob. The authors are indebted to the people of LNE-SYRTE laboratory for the time reference and constant help during all these years.

\section{References}

1. F. Biraben, P. Labastie, Opt. Commun. 41, 49 (1982)

2. T.W. Hänsch, I.S. Shahin, A.L. Schawlow, Nature 235, 63 (1972)

3. T.W. Hänsch, S.A. Lee, R. Wallenstein, C. Wieman, Phys. Rev. Lett. 34, 307 (1975)

4. B. Cagnac, G. Grynberg, F. Biraben, J. Phys. 34, 845 (1973)

5. F. Biraben, B. Cagnac, G. Grynberg, Phys. Rev. Lett. 32, 643 (1974)

6. F. Biraben, E. Giacobino, G. Grynberg, Phys. Rev. A 12, 2444 (1975)

7. E. Giacobino, F. Biraben, J. Phys. B: At. Mol. Phys. 15, L385 (1982)

8. M. Glass-Maujean, L. Julien, T. Dohnalik, J. Phys. B. 11, 421 (1978)

9. T.W. Hänsch, B. Couillaud, Opt. Commun. 35, 441 (1980)

10. F. Biraben, L. Julien, Opt. Commun. 53, 320 (1985)

11. J.E.M. Goldsmith, E.W. Weber, T.W. Hänsch, Phys. Rev. Lett. 41, 1525 (1978)

12. B.W. Petley, K. Morris, R.E. Shawyer, J. Phys. B: At. Mol. Phys. 13, 3099 (1980)

13. S.R. Amin, C.D. Caldwell, W. Lichten, Phys. Rev. Lett. 47, 1234 (1981)

14. C. Foot, B. Couillaud, R.G. Beausoleil, T.W. Hänsch, Phys. Rev. Lett. 54, 1913 (1985)

15. E.A. Hildum, U. Boesl, D.H. McIntyre, R.G. Beausoleil, T.W. Hänsch, Phys. Rev. Lett. 56, 576 (1986)

16. YuL Sokolov, V.P. Yakovlev, Sov. Phys. JETP 56, 7 (1982)

17. S.R. Lundeen, F.M. Pipkin, Phys. Rev. Lett. 46, 232 (1981)

18. F. Biraben, J.C. Garreau, L. Julien, Europhys. Lett. 2(12), 925 (1986)

19. J.C. Garreau, M. Allegrini, L. Julien, F. Biraben, J. Phys. 51, $2275(1990)$

20. F. Biraben, J.C. Garreau, L. Julien, M. Allegrini, Rev. Sci. Instrum. 61, 1468 (1990)

21. F. Biraben, J.C. Garreau, L. Julien, M. Allegrini, Phys. Rev. Lett. 62, 621 (1989)

22. P. Zhao, W. Lichten, H.P. Layer, J.C. Bergquist, Phys. Rev. Lett. 58, 1293 (1987)

23. R.G. Beausoleil, D.H. McIntyre, C.J. Foot, E.A. Hildum, B. Couillaud, T.W. Hänsch, Phys. Rev. A 35, 4878 (1987)

24. M.G. Boshier, P.E.G. Baird, C.J. Foot, E.A. Hinds, M.D. Plimmer, D.N. Stacey, J.S. Swan, D.A. Tate, D.M. Warrington, G.K. Woodgate, Nature 330, 463 (1987)

25. M. Weitz, A. Huber, F. Schmidt-Kaler, D. Leibfried, W. Vassen, C. Zimmermann, K. Pachucki, T.W. Hänsch, L. Julien, F. Biraben, Phys. Rev. A 52, 2664 (1995)

26. D.J. Berkeland, E.A. Hinds, M.G. Boshier, Phys. Rev. Lett. 75, 2470 (1995)

27. M.G. Boshier, P.E.G. Baird, C.J. Foot, E.A. Hinds, M.D. Plimmer, D.N. Stacey, J.B. Swan, D.A. Tate, D.M. Warrington, G.K. Woodgate, Phys. Rev. A 40, 6169 (1989)

28. S. Bourzeix, M.D. Plimmer, F. Nez, L. Julien, F. Biraben, Opt. Commun. 99, 89 (1993)

29. S. Bourzeix, B. de Beauvoir, F. Nez, F. de Tomasi, L. Julien, F. Biraben, Opt. Commun. 133, 239 (1997)

30. S. Bourzeix, B. de Beauvoir, F. Nez, M.D. Plimmer, F. de Tomasi, L. Julien, F. Biraben, Phys. Rev. Lett. 76, 384 (1996)

31. F. Nez, M.D. Plimmer, S. Bourzeix, L. Julien, F. Biraben, R. Felder, Y. Millerioux, P. de Natale, Europhys. Lett. 24, 635 (1993) 
32. F. Biraben, D. Jasmin, L. Julien, F. Nez, J. Vigué, Opt. Commun. 92, 135 (1992)

33. F. Nez, F. Biraben, R. Felder, Y. Millerioux, Opt. Commun. 102, $432(1993)$

34. D. Touahri, O. Acef, A. Clairon, J.J. Zondy, R. Felder, L. Hilico, B. de Beauvoir, F. Biraben, F. Nez, Opt. Commun. 133, 471 (1997)

35. L. Hilico, R. Felder, D. Touahri, O. Acef, A. Clairon, F. Biraben, Europhys. J. Appl. Phys. 4, 219 (1998)

36. B. de Beauvoir, F. Nez, L. Hilico, L. Julien, F. Biraben, B. Cagnac, J.J. Zondy, D. Touahri, O. Acef, A. Clairon, Eur. Phys. J. D 1, 227 (1998)

37. F. Nez, M.D. Plimmer, S. Bourzeix, L. Julien, F. Biraben, Phys. Rev. Lett. 69, 2326 (1992)

38. B. de Beauvoir, F. Nez, L. Julien, B. Cagnac, F. Biraben, D. Touahri, L. Hilico, O. Acef, A. Clairon, J.J. Zondy, Phys. Rev. Lett. 78, 440 (1997)

39. H.R. Telle, D. Meschede, T.W. Hänsch, Opt. Lett. 15, 532 (1990)

40. C. Schwob, L. Jozefowski, B. de Beauvoir, L. Hilico, F. Nez, L. Julien, F. Biraben, Phys. Rev. Lett. 82, 4960 (1999)

41. B. de Beauvoir, C. Schwob, O. Acef, L. Jozefowski, L. Hilico, F. Nez, L. Julien, A. Clairon, F. Biraben, J.J. Zondy, Eur. Phys. J. D 12, 61 (2000)

42. D.H. McIntyre, R.G. Beausoleil, C.J. Foot, E.A. Hildum, B. Couillaud, T.W. Hänsch, Phys. Rev. A 39, 4591 (1989)

43. Th Udem, A. Huber, B. Gross, J. Reichert, M. Prevedelli, M. Weitz, T.W. Hänsch, Phys. Rev. Lett. 79, 2646 (1997)

44. M. Niering, R. Holzwarth, J. Reichert, P. Pokasov, Th Udem, M. Weitz, T.W. Hänsch, P. Lemonde, G. Santarelli, M. Abgrall, P. Laurent, C. Salomon, A. Clairon, Phys. Rev. Lett. 84, 5496 (2000)

45. A. Matveev, C.G. Parthey, K. Predehl, J. Alnis, A. Beyer, R. Holzwarth, T. Udem, T. Wilken, N. Kolachevsky, M. Abgrall, D. Rovera, C. Salomon, P. Laurent, G. Grosche, O. Terra, T. Legero, H. Schnatz, S. Weyers, B. Altschul, T.W. Hänsch, Phys. Rev. Lett. 110, 230801 (2013)

46. J. Reichert, M. Niering, R. Holtzwarth, M. Weitz, Th Udem, T.W. Hänsch, Phys. Rev. Lett. 84, 3232 (2000)

47. M. Fischer, N. Kolachevsky, M. Zimmermann, R. Holzwarth, Th Udem, T.W. Hänsch, M. Abgrall, J. Grnert, I. Maksimovic, S. Bize, H. Marion, F. Pereira Dos Santos, P. Lemonde, G. Santarelli, P. Laurent, A. Clairon, C. Salomon, M. Haas, U.D. Jentschura, C.H. Keitel, Phys. Rev. Lett. 92, 230802 (2004)

48. C.G. Parthey, A. Matveev, J. Alnis, B. Bernhardt, A. Beyer, R. Holzwarth, A. Maistrou, R. Pohl, K. Predehl, Th Udem, T. Wilken, N. Kolachevsky, M. Abgrall, D. Rovera, Ch. Salomon, Ph Laurent, T.W. Hänsch, Phys. Rev. Lett. 107, 203001 (2011)

49. F. Biraben, L. Julien, J. Plon, F. Nez, Opt. Commun. 15, 831 (1991)

50. G. Hagel, R. Battesti, F. Nez, L. Julien, F. Biraben, Phys. Rev. Lett. 89, 203001 (2002)

51. O. Arnoult, F. Nez, L. Julien, F. Biraben, Eur. Phys. J. D 60, 243 (2010)

52. S. Galtier, F. Nez, L. Julien, F. Biraben, Opt. Commun. 324, 34 (2014)

53. S.Galtier, H. Fleurbaey, S. Thomas, L. Julien, F. Biraben, F. Nez, J. Phys. Chem. Ref. Data 44, 031201 (2015)

54. S. G. Karshenboim, J. Phys. B 29, L29 (1996)

55. S.G. Karshenboim, Z. Phys. D 39, 109 (1997)

56. A. Czarnecki, U.D. Jentschura, K. Pachucki, Phys. Rev. Lett. 95, $180404(2005)$
57. P.J. Mohr, D.B. Newell, B.N. Taylor, arXiv:1507.07956v1 (2015)

58. R. Pohl, A. Antognini, F. Nez, F.D. Amaro, F. Biraben, J.M.R. Cardoso, D. Covita, A. Dax, S. Dhawan, L. Fernandes, A. Giesen, T. Graf, T.W. Hänsch, P. Indelicato, L. Julien, C.-Y. Kao, P.E. Knowles, E. Le Bigot, Y.-W. Liu, J.A.M. Lopes, L. Ludhova, C.M.B. Monteiro, F. Mulhauser, T. Nebel, P. Rabinowitz, J.M.F. dos Santos, L. Schaller, K. Schuhmann, C. Schwob, D. Taqqu, J.F.C.A. Veloso, F. Kottmann, Nature 466, 213 (2010)

59. A. Antognini, F. Nez, K. Schuhmann, F.D. Amaro, F. Biraben, J.M.R. Cardoso, D. Covita, A. Dax, S. Dhawan, M. Diepold, L. Fernandes, A. Giesen, A. Gouvea, T. Graf, T.W. Hänsch, P. Indelicato, L. Julien, C.-Y. Kao, P.E. Knowles, F. Kottmann, E. Le Bigot, Y.-W. Liu, J.A.M. Lopes, L. Ludhova, C.M.B. Monteiro, F. Mulhauser, T. Nebel, P. Rabinowitz, J.M.F. dos Santos, L. Schaller, C. Schwob, D. Taqqu, J.F.C.A. Veloso, J. Vogelsang, R. Pohl, Science 339, 417 (2013)

60. R. Pohl, F. Nez, L.M.P. Fernandes, F.D. Amaro, F. Biraben, J.M.R. Cardoso, D.S. Covita, A. Dax, S. Dhawan, M. Diepold, A. Giesen, A.L. Gouvea, T. Graf, T.W. Hänsch, P. Indelicato, L. Julien, P. Knowles, F. Kottmann, E.O. Le Bigot, Y.W. Liu, J.A.M. Lopes, L. Ludhova, C.M.B. Monteiro, F. Mulhauser, T. Nebel, P. Rabinowitz, J.M.F. dos Santos, L.A. Schaller, K. Schuhmann, C. Schwob, D. Taqqu, J.F.C.A. Veloso, A. Antognini, Science 353, 669 (2016)

61. E. Hessels, in Frontiers in Optics 2015. OSA Technical Digest (online) (Optical Society of America, 2015), paper LTu2G.2

62. A. Beyer, J. Alnis, K. Khabarova, A. Matveev, C.G. Parthey, D.C. Yost, R. Pohl, T. Udem, T.W. Hänsch, N. Kolachevsky, Anna. Phys. 525, 671 (2013)

63. E. Peters, D.C. Yost, A. Matveev, T.W. Hänsch, T. Udem, Ann. Phys. 525, L29 (2013)

64. M. Horbatsch, E.A. Hessels, Phys. Rev. A 82, 052519 (2010)

65. D.C. Yost, A. Matveev, E. Peters, A. Beyer, T.W. Hänsch, Th Udem, Phys. Rev. A 90, 012512 (2014)

66. P. Amaro, B. Franke, J.J. Krauth, M. Diepold, F. Fratini, L. Safari, J. Machado, A. Antognini, F. Kottmann, P. Indelicato, R. Pohl, J.P. Santos, Phys. Rev. A 92, 022514 (2015)

67. M. Herrmann, M. Haas, U.D. Jentschura, F. Kottmann, D. Leibfried, G. Saathoff, C. Gohle, A. Ozawa, V. Batteiger, S. Knnz, N. Kolachevsky, H.A. Schssler, T.W. Hänsch, Th Udem, Phys. Rev. A 79, 052505 (2009)

68. U.D. Jentschura, P.J. Mohr, J.N. Tan, B.J. Wundt, Phys. Rev. Lett. 100, 160404 (2008)

69. J.P Karr, L. Hilico, J.C.J. Koelemeij, V.I. Korobov, arXiv:1605.05456 [physics.atom-ph] (2016)

70. R. Pohl, F. Nez, L.M.P. Fernandes, M. Abdou Ahmed, F.D. Amaro, P. Amaro, F. Biraben, J.M.R. Cardoso, D.S. Covita, A. Dax, S. Dhawan, M. Diepold, B. Franke, S. Galtier, A. Giesen, A.L. Gouvea, J. Götzfried, T. Graf, T.W. Hänsch, M. Hildebrandt, P. Indelicato, L. Julien, K. Kirch, A. Knecht, P. Knowles, F. Kottmann, J.J. Krauth, E-O. Le Bigot, Y-W. Liu, J.A.M. Lopes, L. Ludhova, J. Machado, C.M.B. Monteiro, F. Mulhauser, T. Nebel, P. Rabinowitz, J.M.F. dos Santos, J. Paulo Santos, L.A. Schaller, K. Schuhmann, C. Schwob, C.I. Szabo, D. Taqqu, J.F.C.A. Veloso, A. Voss, B. Weichelt, A. Antognini, in Proceedings of LEAP 2016 (submitted to Japanese Physical Society)

71. K.S.E. Eikema, J. Walz, T.W. Hänsch, Phys. Rev. Lett. 86, 5679 (2001)

72. A.L. Schawlow, T.W. Hänsch, G.W. Series, Sci. Am. 240, 94 (1979) 